\title{
Dynamics and monitoring of vibratory piling process
}

\author{
Erno Keskinena, Juha-Matti Kivinen ${ }^{a}$, Juha Miettinen ${ }^{a}$, Michel Cotsaftis ${ }^{b}$ \\ Robert Hildebrand $^{\mathrm{c}}$ \& Yrjö Raunisto ${ }^{\mathrm{d}}$
}

\author{
${ }^{a}$ Tampere University of Technology, Laboratory of Machine Dynamics \\ P.O.Box 589, Fin-33101 Tampere, Finland \\ Fax +358331152307 \\ Email:erno.keskinen@tut.fi \\ ${ }^{b}$ Ecole Centrale d'Electronique \\ Paris, France \\ Email:mcot@ece.fr \\ ${ }^{c}$ The Royal Institute of Technology \\ Stockholm, Sweden \\ Email:robert@fkt.kth.se \\ ${ }^{d}$ Unisto Oy \\ Hämeenlinna, Finland \\ Email:unisto@kolumbus.fi.
}

\begin{abstract}
The foundations of buildings in urban environments are very often piled ones. During earth moving works the sides of digging area have to be supported by sheet pile walls to maintain constant earth pressure conditions against existing foundations. The piles and sheet piles are very often driven by vibratory methods, in which case the ground vibration is a risk for neighboring old buildings. The purpose of the present research work is to find a new, theoretically well established way to use vibratory pile drivers environmentally friendly especially in urban environments. Closely related to this a new electro-hydraulic circuit has been introduced by Unisto company for on-line adjusting of the phase angle between primary and secondary shafts of eccentric masses in the vibratory unit. Furthermore, the control of this angle will be subjected to continuous monitoring from the vibration level of the critical structure to be protected. The control of shafts phase angle is based on separate drive technology, in which the position difference is governed by electrically controlled orifices. Vibration control principle is based on the requirement to stay below required vibration bounds in the working environment. The system is equipped with a vibration sensing box to be fixed on to the protected structure, from which the vibration level information is transmitted to the control box of vibratory unit. The described system is investigated both theoretically and numerically. The theoretical part of the research includes the derivation of complete system equations. In the numerical part of the work the system is coded and simulated by computer to get required information for system modifications and dimensioning. The main objective of these research steps is to evaluate the operation of phase angle adjusting system in order to balance the control of environmental vibrations and machine performance.
\end{abstract}

KEYWORDS: Vibratory pile driving, ground vibrations, fluid power systems

\section{INTRODUCTION}

Construction activity has been continuously increasing in areas where conditions for solid foundations are traditionally poor. Typical soft soils are common on river sides, where the urban settlement is historically very often concentrated. High prizing of land has increased the construction efficiency, the consequence of which is the design of tall and heavily founded buildings. The mostly used techniques are to cast pillars on site or to hit piles. Because both new constructions as well as rebuild structures are very often located between existing buildings, the foundation works have strong effect on the stability of neighboring underground structures. A very common solution is to isolate the area to be excavated by sheet pile walls in order to maintain the lateral earth pressure against the existing foundations. The wall consists of an assemblage of separate sheet pile elements locked to each other, each of which has to be hit in to the ground before the start of excavation works. The drive of sheet piles or foundation piles is mostly done in urban environments by vibratory methods instead of noisy hammering method. Vibratory driving method generates propagating 


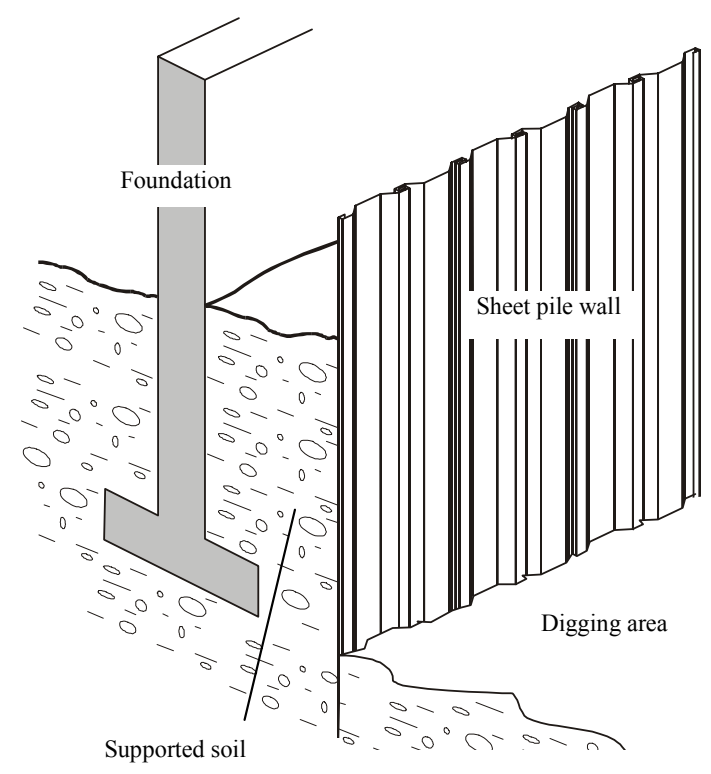

Figure 1. Sheet pile wall supporting existing foundations.

ground vibrations, which are transmitted to the neighboring buildings especially during speed up and slow down [Reusch]. In the worst situation the buildings may get displacements and cracks, both phenomena which are forbidden in the case of historically valuable buildings. Transmission of vibration to offices and apartment houses is also unwanted as it may cause disturbances and damages. Investigations have shown that high intensity vibrations at some vibration speed ranges are related to the passage of critical speeds of the pile - soil - building system.

Vibratory units typically consist of a pair of shafts driven by electrical or fluid power, onto which the eccentric masses are mounted to generate the vertical harmonic force excitation (figure 2). When applying a static driving force the pile penetrates into the ground with a speed depending on frequency and amplitude of the vibration.

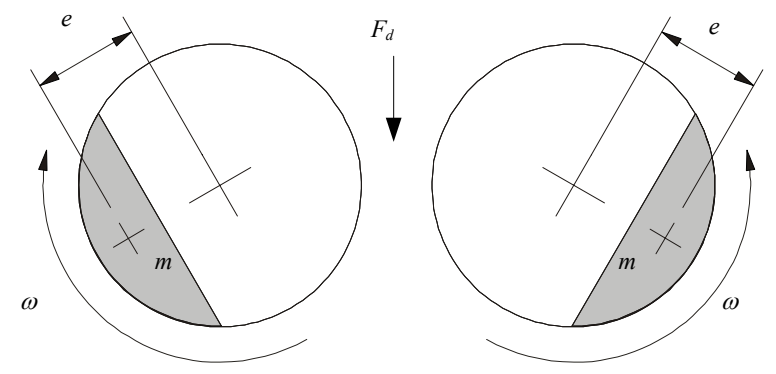

Figure 2. Shaft pair of the vibratory pile driving unit.
The capacity of the unit may be multiplied by adding a number of shaft pairs. This brings the possibility to adjust the phase angle between the eccentric masses in order to continuously add or subtract the component forces. There exist few known mechanisms, with which the phase shift or the mass eccentricity can be varied in a desired way during the drive, thus making it possible to reach resonance-free up and down slides of the speed. One possibility is to use an additional torque motor assembled into the secondary shaft pair to move the angular position of the secondary shaft with respect to the primary one (figure 3). Another way is to vary mass eccentricity by a linear actuator moving the mass in radial direction.
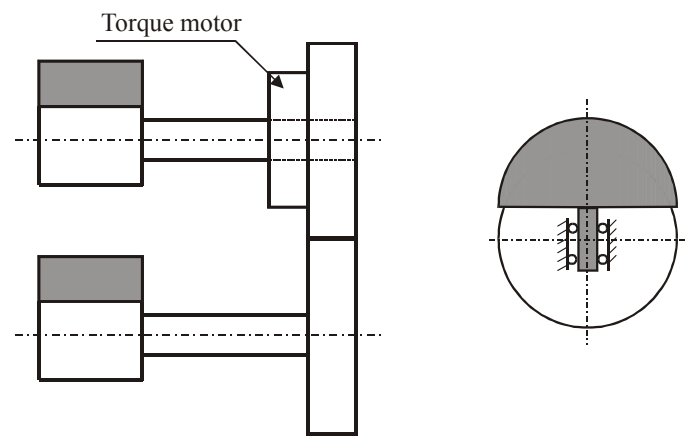

Figure 3. Different possibilities to vary the centrifugal force of the vibratory unit.

Because centrifugal forces are quite large, the actuators installed in the rotating coordinate system need high power density as well. This can be produced by high pressure fluid power cylinders requiring rotating fluid connectors for power supply. The weak point of these arrangements is the frequent maintenance needs at the dynamic seals.

To overcome these problems a new electrohydraulic way has been introduced and patented to control the vibratory driving process by two parameters, the speed of the primary shaft and the phase angle between the primary and secondary shafts. It has been proposed that the operator will adjust these parameters during the run. Because the interaction dynamics of mechanical, structural and hydraulic sub-systems is very complex, the industrial unit has to be equipped with an automatic operation monitoring system. The purpose of the paper is to investigate this solution in order to evaluate its feasibility to real working conditions. 


\section{SYSTEM DESCRIPTION}

The adjustment circuit of the phase shift is based on the solution where eccentric shaft pair numbers 1 (the primary) and 2 (the secondary) are driven by separate hydraulic motors. The shaft pairs are mechanically connected to each other by a gear forcing them to rotate always at the same speed. The gear has a mechanical dead-zone of 180 degrees. If shaft pair 2 is actively pulling only and

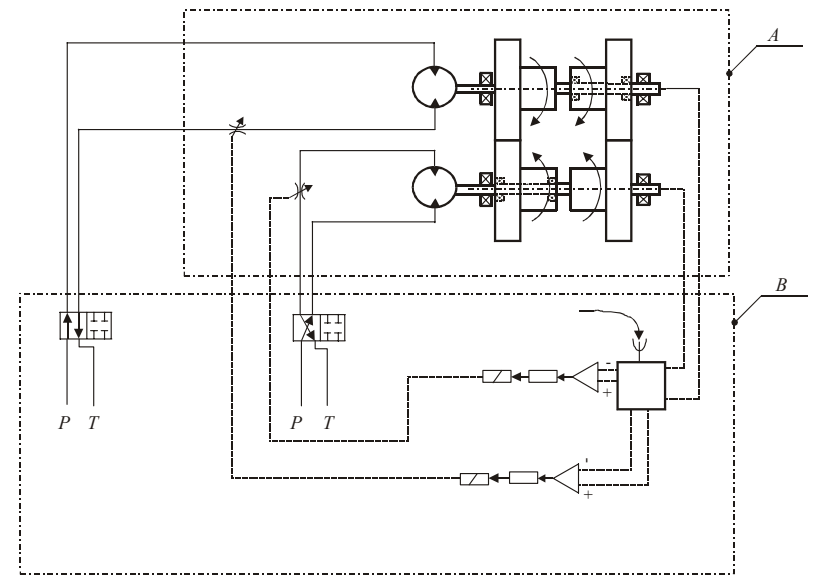

Figure 4. Fluid power circuit for power division.

shaft pair 1 is passively resisting the shafts are in full 180 degrees phase shift and the resulting vertical vibratory force is zero. On the other hand, when shaft number 1 is pushing only and shaft number 2 is resisting the phase angle is zero and the resulting vertical force is the direct sum of the component forces. Intermediate values are reached by combining the pushing torque of shaft 1 and the pulling torque of shaft 2 in a desired ratio. Because both shaft pairs are rotating with the same speed the only physical quantities which indicate the power taken from each motor are the line pressures. The phase angle is controlled by electrically governed valves, which actually work as elements dividing the hydraulic power between the motors. The reduction factor of the resulting vibratory force for each phase shift angles $\Delta \phi=\phi_{2}-\phi_{1}$ is given by

$$
\frac{R}{2 F}=\frac{\sqrt{1+\cos \Delta \phi}}{\sqrt{2}}
$$

A vibratory unit, with on-line adjustable vibration frequency and magnitude, can be flexibly linked to an operation monitoring system. This controls the running parameters based on information measured from the critical structure subject to vibrations (figure 5).

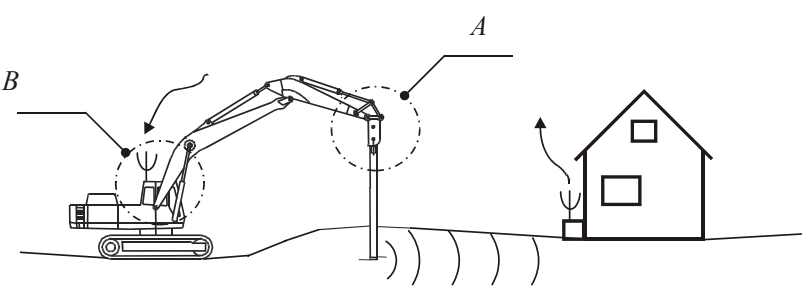

Figure 5. Wireless transmission of ground vibration information to the monitoring system of the piling process.

In normal running conditions the system gives its full power with the exception that during starting speeding up and slowing down the phase angle is full 180 degrees. The process can be run by a sequence control approach consisting of a set of commands to switch proper ramp functions from the control electronics of hydraulic valves.

Furthermore, the monitoring system has a driving control block, which includes a rule basis to process the estimates for proper phase shift value from the measured vibration response.

\section{SYSTEM DYNAMICS}

In order to analyze its behavior before building a physical prototype, the system has been modeled and simulated. The lay-out of the system is shown in figure 6 .

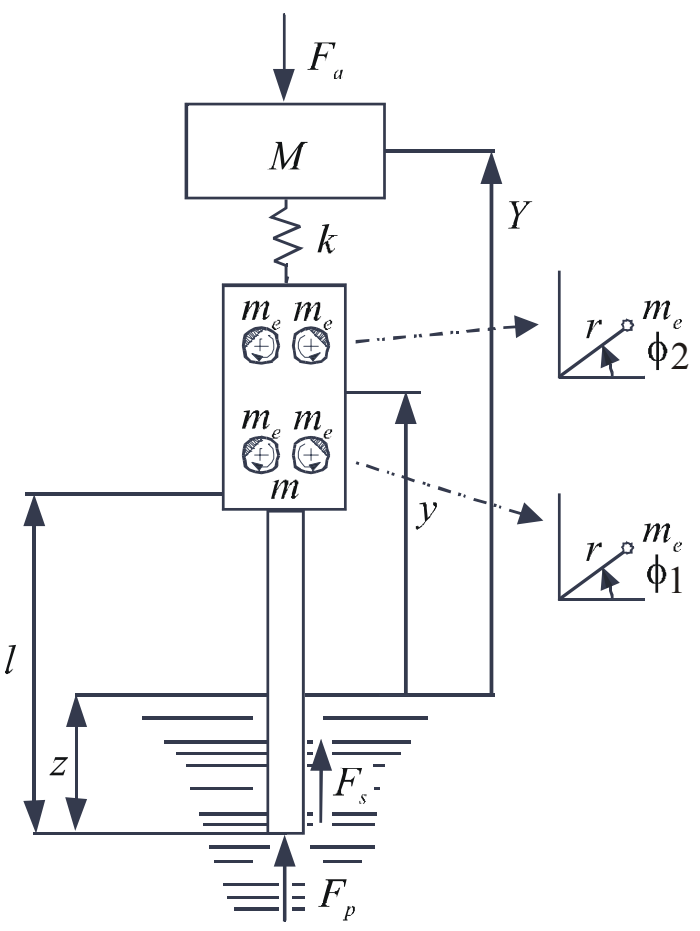

Figure 6. Mechanical model of the four shaft vibratory unit. 
The system degrees of freedom are the vertical position $y$ of vibratory unit and pile combination, the vertical position $Y$ of the frame and the angular positions of mass eccentricities of the shafts. The mathematical model of the mechanical sub-system consists of a group of 4 second order differential equations

$$
\begin{aligned}
& M \ddot{Y}+k(Y-y)=-M g-F_{a} \\
& m^{\prime} \ddot{y}+\mu_{1} c_{1} \ddot{\phi}_{1}+\mu_{2} c_{2} \ddot{\phi}_{2}+k(y-Y)=\mu_{1} s_{1} \dot{\phi}_{1}^{2}+\mu_{2} s_{2} \dot{\phi}_{2}^{2} \\
& \quad-m^{\prime} g-F_{s} \operatorname{sgn}(\dot{y})+F_{p}
\end{aligned}
$$

$I_{1}^{\prime} \ddot{\phi}_{1}+\mu_{1} c_{1} \ddot{y}+\Psi\left(\phi_{1}-\phi_{2}\right)=-g \mu_{1} c_{1}+T_{1}$

$I_{2}^{\prime} \ddot{\phi}_{2}+\mu_{2} c_{2} \ddot{y}-\Psi\left(\phi_{1}-\phi_{2}\right)=-g \mu_{2} c_{2}+T_{2}$

where $c_{i}=\cos \phi_{i}$ and $s_{i}=\sin \phi_{i}$ and $F_{a}, F_{s}$ and $F_{p}$ stand for the feeding force, skin friction and point load of the pile [Launis]. Mechanical coupling of primary and secondary shafts is described by the dead-zone function

$$
\Psi(x)= \begin{cases}K x & ; \quad x<0 \\ 0 & ; \quad 0<x<\pi \\ K(x-\pi) & ; \quad x>\pi\end{cases}
$$

On the other hand, the hydraulic torques generated by the motors are given by

$$
T_{i}=D\left(p_{i}^{p}-p_{i}^{m}\right)
$$

The pressures of driving (p) and resisting (m) chambers are respectively

$$
\begin{aligned}
& \dot{p}_{i}^{p}=\frac{q_{i}^{p}-D \dot{\phi}_{i}}{V} B \\
& \dot{p}_{i}^{m}=\frac{q_{i}^{m}+D \dot{\phi}_{i}}{V} B
\end{aligned}
$$

where the valve flows are

$$
\begin{aligned}
& q_{i}^{p}=c u_{i} \sqrt{p_{s}-p_{i}^{p}} \\
& q_{i}^{m}=c u_{i} \sqrt{p_{i}^{m}-p_{t}}
\end{aligned}
$$

in which the $u_{i}$ 's are the control inputs to the proportional flow control valves. Their current values are output from the vibration control block system.

\section{SYSTEM MODEL}

Analysis of the system can be carried out by means of numerical time integration. Because the elements and components in the system represent quite common parts in mechatronic systems, the system model can be built from existing library objects. Utilization of a modeling environment developed at Machine Dynamics Laboratory in Tampere University of Technology led to the system composition illustrated in figure 7 .

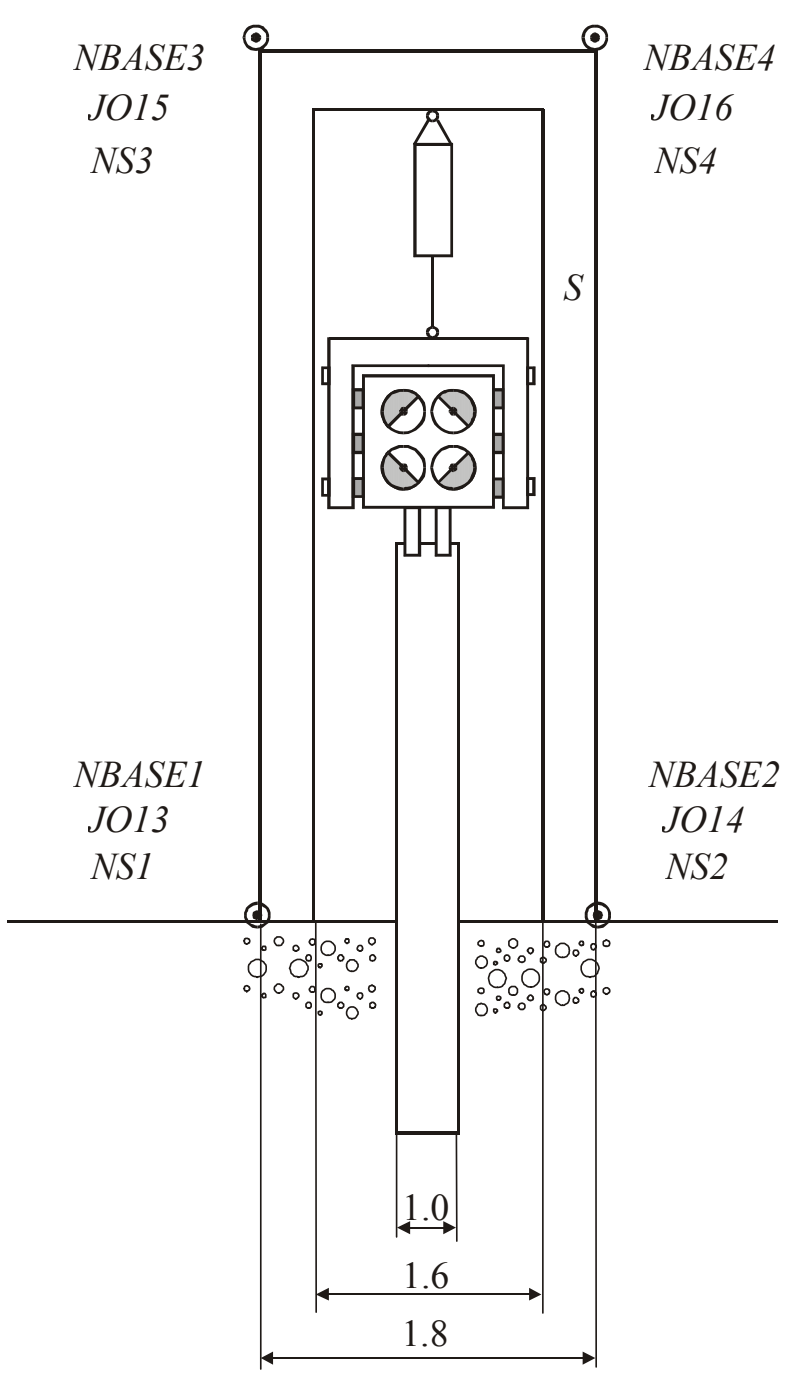

Figure 7. System composition in the objectoriented modeling environment.

In order to keep pile vertical trajectory, the vibratory unit is horizontally locked in a vertical slider, which arrangement serves the modeling task only. The vibratory unit consists of submodels for the eccentric shafts, for the frame and for the isolation rubber springs (figure 8). 


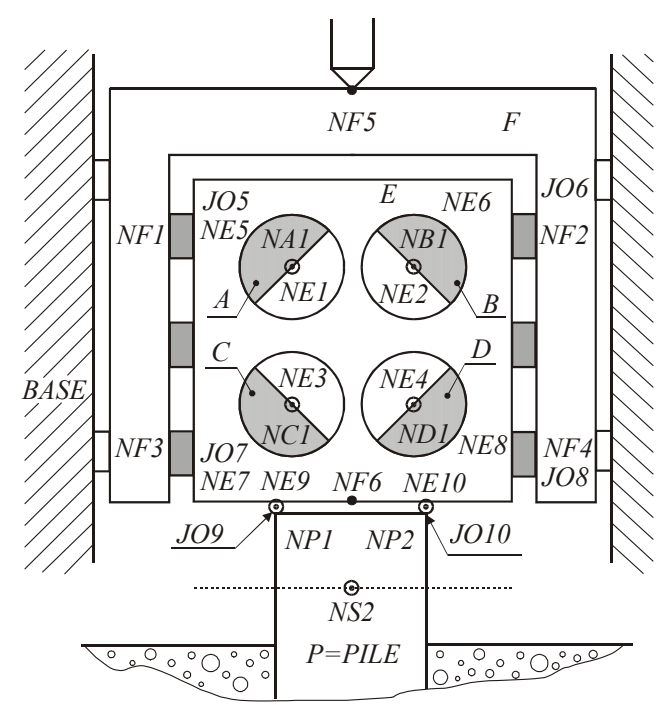

Figure 8. Component model of the vibratory unit.

The fluid power system is a composition of standard component models for valves and motors linked by fluid volumes in the pipes (figure 9).

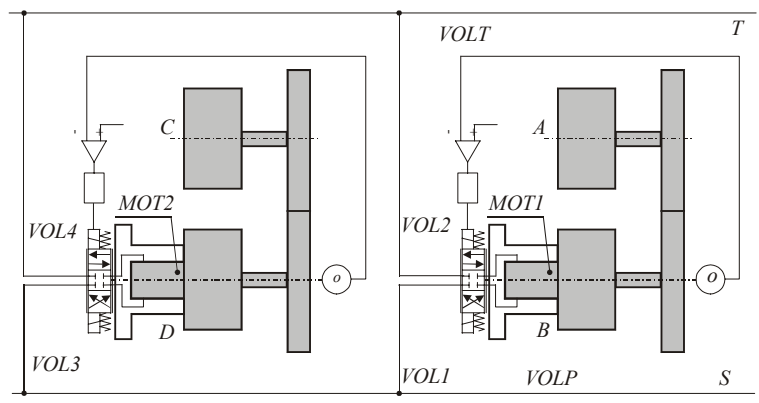

Figure 9. Sub-model of the fluid power circuit.

The complete system model is an order independent list of calls to component models. This reads in Fortran 95 standard format as given below:

SUBROUTINE MOVAXPILER (M NAME, MEC, ARM, JOINT, GEAR, CYL, MOT, VAL, RAMP , PID , VOL, PRES)

C Definitions for component structures RECORD /MEC STRUCT/ MEC

RECORD /ARM STRUCT/ ARM(8)

RECORD /CONT STRUCT/ JOINT (12)

RECORD / GEAR STRUCT/ GEAR (3)

RECORD /HYMOT_STRUCT/ MOT (2)

RECORD / HYCYL STRUCT/ CYL

RECORD /VOLUME_STRUCT/ VOL (8)

RECORD /DCV STREUCT/VAL (3)

RECORD /CTRL STRUCT/ PID(3)

RECORD /SEQ_STRUCT/ RAMP (3)

C Guiding frame

CALL MECBASE (M NAME, "BASE", MEC, ARM (1))

C Vibratory unit

CALL MECARM (M NAME, "E", MEC, ARM (2))

C Eccentric shafts

CALL MECBODY (M NAME, "A", MEC, ARM (3))

CALL MECBODY (M NAME, "B", MEC, ARM (4))

CALL MECBODY (M_NAME, "C", MEC, ARM (5))

CALL MECBODY (M NAME, "D", MEC, ARM (6))

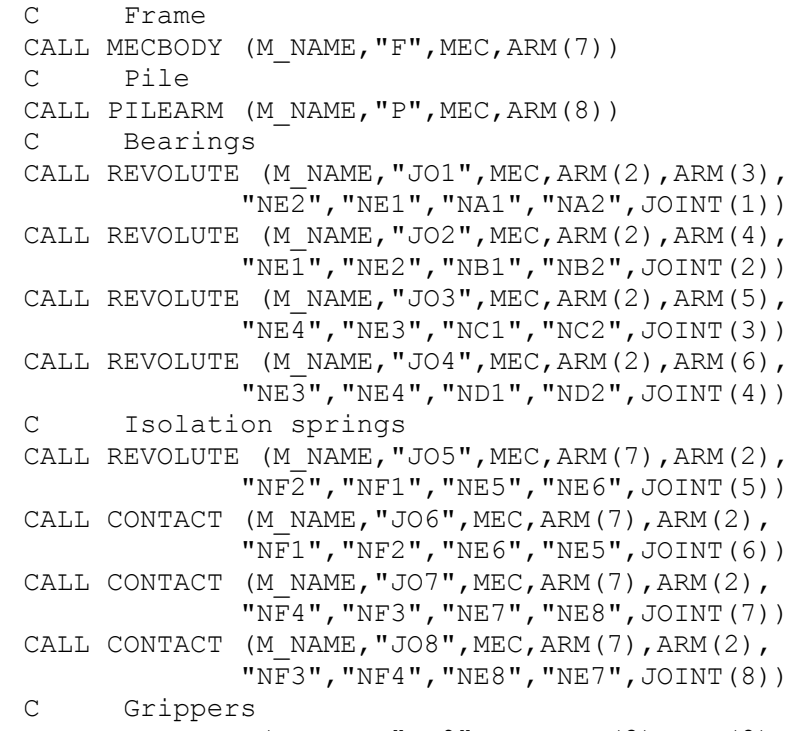

CALL REVOLUTE (M_NAME, "JO9", MEC,ARM (2), ARM (8), "NE10", "NE9", "NP1", "NP2", JOINT (9))

CALL CONTACT (M_NAME, "JO10", MEC, ARM (2), ARM (8), "NE9", "NE10", "NP2", "NP1", JOINT (10) )

C Guiding slider

CALL INTSLIDE (M_NAME, "JO11", "Jo12", MEC, $\operatorname{ARM}(1), \bar{A} R M(7)$, "NBASE1", "NBASE2", "NF5", "NF6", JOINT (11), JOINT (12)) C Gears

CALL GEARTRA (M NAME, "GEARI", MEC, ARM (3), ARM (4), GEAR (1), 0.D0, 0.D0, 0.D0)

CALL GEARTRA (M NAME, "GEAR2", $\operatorname{MEC}, \operatorname{ARM}(5), \operatorname{ARM}(6)$, GEAR (2), 0.D0, 0.D0, 0.D0)

CALL GEARTRA (M_NAME, "GEAR3", MEC, $\operatorname{ARM}(3), \operatorname{ARM}(6)$, GEAR (3), 0.DO, 0.DO, PI)

C Sequence control

CALL DCV RAMP (M NAME, "DCV1", RAMP (1), VAL (1)) CALL DCV_RAMP (M-NAME, "DCV2", RAMP (2), VAL (2)) C Hydráulic motors

CALL HYDMOT (M NAME, "MOT1", MEC, $\operatorname{ARM}(2), \operatorname{ARM}(4)$, "Nㅡ2", "NB1", VOL (1), VOL (2), MOT (1))

CALL HYDMOT (M NAME, "MOT2", MEC, ARM (2), ARM (6), " $\mathrm{N} E 4$ ", "ND1", VOL (3), VOL (4), MOT (2))

C Hydraulic volumes

CALL HYDVOL (M_NAME, "VOL1", MEC, VOL (1))

CALL HYDVOL (M-NAME, "VOL2", MEC, VOL (2))

CALL HYDVOL (M_NAME, "VOL3", MEC, VOL (3))

CALL HYDVOL (M NAME, "VOL4", MEC, VOL (4))

CALL HYDVOL (M-NAME, "VOL5", MEC, VOL (5))

CALL HYDVOL (M_NAME, "VOLP", MEC, VOL (7))

CALL HYDVOL (M- NAME, "VOLT", MEC, VOL (8))

C Proportional valves

CALL SRV (M_NAME, "SRV1", MEC, VOL (7), VOL (8), VOL (1), VOL (2), VAL (1))

CALL SRV (M_NAME, "SRV2", MEC, VOL ( 7), VOL ( 8 ) , VŌL (3), VOL (4), VAL (2))

RETURN

END

\section{CASE STUDY}

The system model has been used for feasibility studies of the introduced vibration control system. The case study consists of runs, in which the performance of the phase shift control has been compared without and with the connection gear.

In the first case the primary shaft pair is working as the "master" while the secondary "slave" shaft is trying to keep the phase shift as constant. 


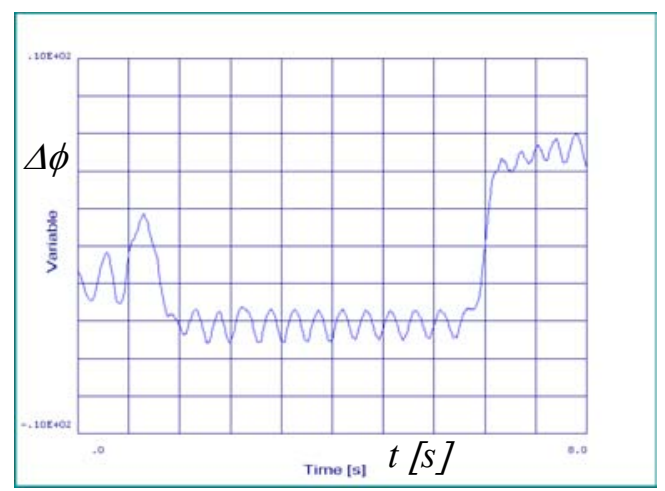

Figure 9. Phase shift of shaft pairs for continuous feedback control of the secondary shaft.

The response of the phase shift is given in figure 9. The response behavior shows that the dynamic interaction of the shaft pairs mounted on the same frame body is so strong that classical PID type controls are not able to keep the phase shift in the neighborhood of the desired value.

In the second case the connection gear has been used under independent sequence control of the shaft pairs. The response plot of the phase shift (figure 10) shows regular behavior in changes from full amplitude reduction, phase shift $=180$ degrees, to maximum amplitude, phase shift $=0$.

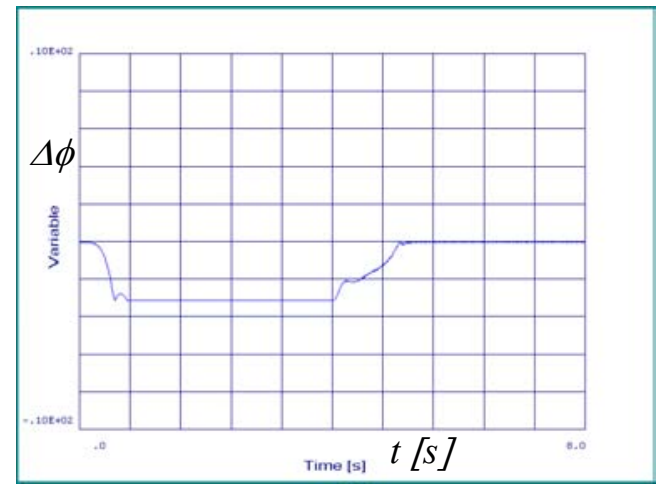

Figure 9. Phase shift between shaft pairs for open loop control of the gear-connected shaft lines.

The result shows that the mechanical dead zone works well together with the sequence control of the shafts. The lower and upper bound values of the dead zone are reached in the desired way. Thus resonance-free speed slides as well as maximum pile driving performance are available in a robust way. Continuous control of the intermediate values is, however, a much more challenging task, which has been left to the subject of further developments.

\section{CONCLUSIONS}

Even if by far less harmful to environment than hammering methods, the usual vibratory driving methods are still generating propagating ground vibrations which, especially during speed up and slow down phases, do not meet new restrictive constraints imposed for respecting urban environment. There is a further need to master vibration dynamics and particularly resonance crossing. This can be dealt with by using a new patented two parameter electro-hydraulic way for controlling vibratory driving process, where the parameters are respectively the speed of primary shaft and the phase angle between the two shafts. The dynamical equations representing this system have been set down, which includes the three mechanical, structural and hydraulic sub-systems and their interactions. To analyse the system and its feasibility in real world situation, a preliminary case has been studied which deals with the sensitive question of the coupling control of the two shaft pairs. The results are showing that in open loop case (i.e. independent sequence control of shaft pairs), the upper and lower bounding values of the dead zone are approached in desired way which does not limit pile driving performances. On the other hand, in closed loop case where the second shaft line is feedback controlled, maintaining the phase shift between shaft pairs to a constant value is not possible here for simple PID controller, and always leads to oscillatory motion around nominal values. This indicates the needs for more adapted controller to better manage the interaction between shaft pairs.

\section{REFERENCES}

[Reusch] Reusch, D., Automatic controlled vibratory pile driving with schock stress limitation. Proceedings of 17th International Symposium on Automation and Robotics in Construction, September 18-20, 2000, Taipei, Taiwan.

[Launis] Launis, S., Keskinen, E.K. \& Raunisto, Y., Dynamic Analysis of Pile Penetration During Vibratory Driving - A Comparative Study, of Linear and Nonlinear Models, Proceedings of the IffToMM 10th World Congress on the Theory of Machines and Mechanisms, Oulu, Finland, June 20-24, 1999, pp. 1631-1637. 